\title{
Fading Channel with Orthogonal Multi-carrier Code Division Multiple Access Signal
}

\author{
Issam Trrad ${ }^{1}$, Amjad Hindi ${ }^{1}, Z_{i a d}$ Sobih $^{2, *}$ \\ ${ }^{1}$ Department of Electrical Engineering, Jadara University, Irbid, 21110, Jordan \\ ${ }^{2}$ Department of Electrical Engineering, Isra University, Amman, 11622, Jordan
}

\begin{abstract}
The wireless industry is currently undergoing a major transition from second generation $(2 \mathrm{G})$ to third generation $(3 \mathrm{G})$ wireless technologies, and the increasing demands for communication services require higher transmission rates that further stimulates the demand on wideband technologies depicted in the form of code division multiple access (CDMA) on one hand. Also, as the privacy is a prime issue in modern communications therefore the carriers orthogonality is a fundamental concept for a sustainable development of the communications sector. This paper attempts to assess wireless communications channels and propose techniques to improve the performance of modern wireless communications. Hence from a strategic perspective, an analytical study into the performance of Group Orthogonal Multi Carrier Code Division Multiple Access (GO-MC-CDMA) in which subcarriers assigned to users which are orthogonal to other groups, the channel bandwidth is the main theme of this paper; from which the problems that may affect the communications link are high lighted and measures to counterbalance and alleviate multipath fading are proposed and investigated. Such measures are: Space diversity combining techniques and minimum - mean square error, and interference cancellation and detection are considered in this research. As a result of this research it can be stipulated that subcarriers grouping does reduce Multi-User-Interference (MUI) between frequency groups thus enhance the performance of MC-CDMA system in the mission of meeting newly emerging wideband communications services, such as video conference and others.
\end{abstract}

Keywords Fading Channel, Code Division Multiple Access (CDMA), Multi User Interference, Additive White Gaussian Noise, Frequency Division Multiple Access, Multiuser Orthogonal Frequency Division Multiplexing (M-OFDM)

\section{Introduction}

OFDM is a robust scheme to frequency selective fading, however, it has several disadvantages such as difficulty in subcarrier synchronization and sensitivity to frequency offset and nonlinear amplification, which result from the fact that it is composed of a number of subcarriers with their overlapping power spectra and exhibits a non-constant nature in its envelope. However, the combination of OFDM signaling and CDMA scheme has one major advantage that it can lower the symbol rate in each subcarrier so that a longer symbol duration makes it easier to quasi-synchronize the transmissions. The Multicarrier CDMA schemes are categorized mainly into two groups: One spreads the original data stream using a given spreading code, and then modulates a different subcarrier with each chip (in a sense that the spreading operation is in the frequency domain), and other spreads the serial-to-parallel (S/P); converted data streams using a given spreading code, and then modulates a

* Corresponding author:

abood201140@yahoo.com (Ziad Sobih)

Published online at http://journal.sapub.org/ijnc

Copyright (C) 2011 Scientific \& Academic Publishing. All Rights Reserved different subcarrier with each of the data stream (the spreading operation in this is in the time domain); similar to a normal direct sequence code division multiple access (DS-CDMA) scheme[1]. In order to exploit the maximum possible channel diversity while being able to accommodate dynamic load changes, a group orthogonal multi carrier code division multiple access (GO-MC-CDMA) scheme is developed. This scheme does not require complex code assignment operations. The set of subcarriers is partitioned into groups, and the users who are assigned subcarriers of the same group are separated using spreading codes. Furthermore, it can be seen that grouping the subcarriers together will help the user to achieve full multipath diversity which is essential for the mitigation of the multipath effects. Also the users in each group are immune to interference from other groups, hence explains the name of this scheme, as group orthogonal MC-CDMA.

\section{System Model}

In this section a complete modeling of the above system is presented. A symbol-spread case is considered, where each active user transmits only one symbol over a block of $\mathrm{N}_{\mathrm{c}}$ chips, the symbol period is $\mathrm{T}=\mathrm{N}_{\mathrm{c}} \mathrm{T}_{\mathrm{c}}$. The entire availa- 
ble bandwidth is utilized with $\mathrm{N}_{\mathrm{c}}$ subcarriers that are spaced by $1 / T$ apart. If $\mathbf{f}_{\mathbf{i}}$ denotes the ith column of the Fast Fourier Transform (FFT) matrix $\mathbf{F}_{\mathrm{Nc}}$, then $\mathbf{f}_{\mathbf{i}}^{*}$ is the ith digital subcarrier. The $\mathrm{N}_{\mathrm{c}}$ subcarriers are partitioned into $\mathrm{Ng}$ groups with each group having $\mathrm{Q}=\mathrm{N}_{\mathrm{c}} / \mathrm{Ng}$ subcarriers. A user chooses a specific group of subcarriers to transmit its information bearing symbols; and Q users share Q subcarriers per group, which ensures no spectral efficiency loss[2]. The system model of GO-MC-CDMA is illustrated in the block diagram of Figure 1. Let $S_{n, m}(i)$ be the information bearing symbol of user $m$ in the nth group transmitted during the time interval $[\mathrm{iT},(\mathrm{i}+1) \mathrm{T})]$.

$\mathrm{A} Q \times 1$ spreading code $\mathrm{cm}$ is used to spread $\mathrm{Sn}, \mathrm{m}(\mathrm{i})$ to the $\mathrm{Q}$ subcarriers of the nth group. the $\mathrm{Q} \times \mathrm{Q}$ matrix is defined as $\mathbf{C}:=\left[\mathbf{c}_{0}, \mathbf{c}_{1}, \ldots, \mathbf{c}_{\mathrm{Q}-1}\right]$, whose columns consist of $\mathrm{Q}$ spreading codes of the nth group. The spreading code matrix $\mathbf{C}$ does not have to be identical for different groups. But since there is no MUI between users of different groups by design, the same code matrix is chosen for all groups. $C$ is designed such that, all user codes are linearly independent, with $\left|\mathrm{c}_{\mathrm{q}}(\mathrm{i})\right|^{2}=$ $1 / \mathrm{Q}, \forall \mathrm{q}, \mathrm{i}=1, \ldots, \mathrm{Q}$, where $\mathrm{c}_{\mathrm{q}}(\mathrm{i})$ is the ith entry of $\mathrm{c}_{\mathrm{q}}$. This design condition is satisfied when $\mathrm{c}_{\mathrm{q}}$ is a scaled binary code e.g., Walsh-Hadamard or Gold Code or, any constant modulus complex field code.

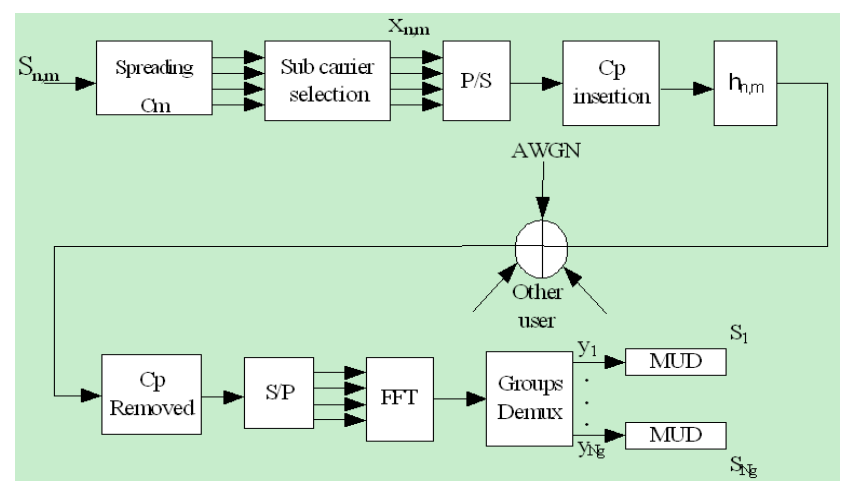

Figure 1. System model of GO-MC-CDMA.

Let the columns of the $\mathrm{N}_{\mathrm{c}} \times \mathrm{Q}$ matrix $\mathrm{F}_{\mathrm{n}}^{*}$ comprise of the $\mathrm{Q}$ digital subcarriers of the nth group, while any $\mathrm{Q}$ subcarriers can be assigned to a group of users, then if a set of equi-spaced subcarriers is selected, the following matrix is obtained:

$$
\mathbf{F}_{\mathbf{n}}^{*}=\left[\mathrm{f}_{\mathrm{n}}^{*}, \mathrm{f}_{\mathrm{Ng}+\mathrm{n}}^{*}, \mathrm{f}_{2 \mathrm{Ng}+\mathrm{n}}^{*}, \ldots . \mathrm{f}_{(\mathrm{Q}-1) \mathrm{Ng}+\mathrm{n}}\right]
$$

where $*$ denotes complex conjugate. This group of subcarrier assignment is illustrated in Figure 2 with $\mathrm{Nc}=9, \mathrm{Ng}=3$, and $\mathrm{Q}=3$.

Where The $\mathrm{N}_{\mathrm{c}} \times 1$ signal vector of user $\mathrm{m}$ in the nth group during the ith block, modulated on $\mathrm{Q}$ subcarriers, may be expressed as

$$
\mathbf{X}_{\mathrm{n}, \mathrm{m}}(\mathrm{i})=\mathbf{F}_{\mathrm{n}}^{*} \mathrm{c}_{\mathrm{m}} \mathrm{S}_{\mathrm{n}, \mathrm{m}}(\mathrm{i})
$$

After parallel-to-serial (P/S) conversion, a cyclic prefix (CP) chips are added to each block, and the signal is transmitted over a frequency-selective fading channel. Notice that $\mathbf{X}_{\mathrm{n}, \mathrm{m}}(\mathrm{i})$ in equation (2) can be computed using a Q-point FFT, and $\mathrm{N}_{\mathrm{c}}+1$ complex multiplications, which considerably reduces the complexity (especially with $\mathrm{N}_{\mathrm{c}}$ large) when compared to the case where $\mathrm{Q}$ subcarriers in a group are arbitrarily chosen. At the receiver end, after removing the $\mathrm{CP}$ to eliminate interblock interference (IBI), and FFT processing the IBI-free signal, the received samples belonging to different groups of subcarriers are demultiplexed.

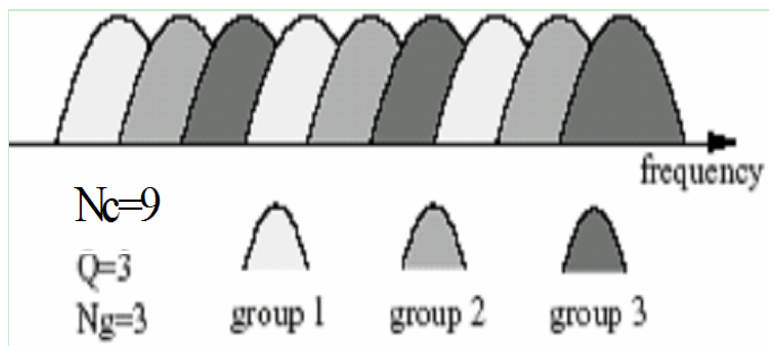

Figure 2. Equi-spaced group subcarrier assignment.

Let $\mathrm{h}_{\mathrm{n}, \mathrm{m}}=\left[\mathrm{H}_{\mathrm{n}, \mathrm{m}}\left(\mathrm{e}^{-\mathrm{j} 2 \pi \mathrm{n} / \mathrm{Nc}}\right), \ldots, \mathrm{H}_{\mathrm{n}, \mathrm{m}}\left(\mathrm{e}^{-\mathrm{j} 2 \pi(\mathrm{n}+(\mathrm{Q}-1) \mathrm{Ng} / \mathrm{Nc}}\right)\right]$ contain the frequency response samples on the FFT grid of the FIR channel of the mth user in the nth group. The $\mathrm{Q} \times 1$ data vector for the nth group can be written as[2].

$$
\mathrm{y}_{\mathrm{n}}=\sum_{\mathrm{m}=0}^{\mathrm{N}_{\mathrm{a}, \mathrm{n}}^{-1}} \mathrm{D}\left(\mathrm{h}_{\mathrm{n}, \mathrm{m}}\right) \mathrm{c}_{\mathrm{m}} \mathrm{S}_{\mathrm{n}, \mathrm{m}}+\mathrm{w}_{\mathrm{n}}
$$

Where $\mathrm{N}_{\mathrm{a}, \mathrm{n}}$ is the number of active users in the nth group, $D(\mathrm{x})$ stands for a diagonal matrix with $\mathrm{x}$ on its diagonal and $\mathrm{W}_{\mathrm{n}}$ is zero-mean complex additive white Gaussian noise (AWGN) with variance $\mathrm{N}_{0} / 2$ per dimension. Since there is no IBI. Based on $\mathrm{y}_{\mathrm{n}}$ in eq.(3), and since there is no IBI, then MUD can be applied to detect the information-bearing symbols $\left\{\mathrm{S}_{\mathrm{n}, \mathrm{m}}\right\}_{\mathrm{m}=0}^{\mathrm{N}_{\mathrm{a}, \mathrm{n}}-1}$ in the nth group. In order to reduce the loss in bandwidth and power efficiency due to $\mathrm{CP}$, the block spreading approach can be incorporated, where $\mathrm{K}>1$ symbols are transmitted per user per block. The $\mathrm{KN}_{\mathrm{c}}$ subcarriers are used to transmit a total of $\mathrm{KN}_{\mathrm{c}}$ symbols per block without increasing signal bandwidth. The subcarriers are partitioned into $\mathrm{Ng}=\mathrm{KN}_{\mathrm{c}} / \mathrm{Q}$ groups. Each user is assigned to $\mathrm{K}$ groups, and transmits one symbol in each group. At the receiver, using the $\mathrm{K}$ input-output Equation (2), the $\mathrm{K}$ symbols are detected of the same user independently. This block spreading of GO-MC-CDMA avoids ISI among symbols from the same user, which facilitates the accommodation of the dynamic load changes in the system to improve the performance at low load[2].

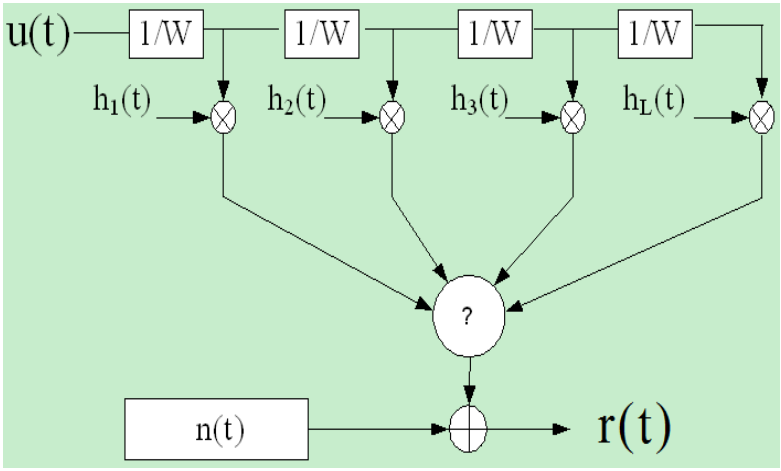

Figure 3. Tapped delay line model of frequency selective channel. 


\section{Fading Channel Model}

The tapped delay line is shown in Figure 3. The time variant tap weights $\left\{h_{p}(\mathrm{n})\right\}$ are zero mean complex valued stationary Gaussian random process, and they correspond to the $\mathrm{L}$ different delays $\tau=\mathrm{pT}_{\mathrm{m}}$, where $\mathrm{p}=1,2, \ldots \mathrm{L}$.

For all practical purposes, the channel tapped delay line model can be truncated at $\mathrm{L}=\left[\mathrm{T}_{\mathrm{m}} \mathrm{W}\right]+1$ taps , where $\mathrm{T}_{\mathrm{m}}$ is a total multipath delay spread and $\mathrm{W}$ is the signal bandwidth. Then, the noisy received signal can be expressed in the form [3]:

$$
r(t)=\sum_{p=1}^{L} h_{p}(t) u\left(t-\frac{p}{W}\right)+n(t)
$$

\section{Space Diversity Technique and Simulation Results}

Figure 4 shows the configuration of a two-branch diversity system. Both branches are weighted by their respective instantaneous voltage-to-noise ratios, the branches are then co-phased prior to summing in order to ensure that all branches are added in phase for maximum diversity gain. The summed signals are used as the received signals, connected then to the demodulator.

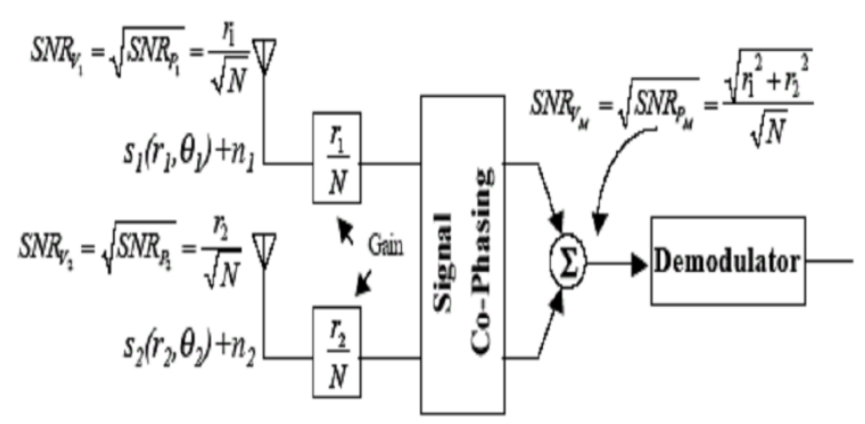

Figure 4. Block diagram of a two-branch maximal ratio combiner for equal noise powers.

However, the disadvantage of MRC; it is a complicated technique that requires accurate estimates of the instantaneous signal level and average noise power to achieve optimum performance. The advantage on the other hand is the improvement that can be achieved with this configuration even when both branches are completely correlated.

Figure $5 \mathrm{c}$ shows the effect of using MRC combining with different number of branches. As the number of branches is increased, the bit error rate decreases, and the complexity of system becomes expensive.

Figure 5d shows the bit error rate (BER) versus signal to noise ratio (SNR) of GO-MC-CDMA system using different detectors. The three stages PIC detector gives a better BER performance, while SIC detector is reducing the error rate at SNR values (7-11) dB.

In order to demonstrate the numerical results, assume that Data rate 3 Mbps (BSK Modulation), Walsh code with length $\mathrm{K}=32$, No. of subcarriers $\mathrm{Nc}=256$, No. of active users $\mathrm{Na}=4$, Delay spread $\mathrm{Td}=20 \mathrm{~ns}$ (Two path), Coherence time $=0.167 \mathrm{~ms}$, Doppler shift $\mathrm{fd}=10 \mathrm{KHz}$, Cyclic prefix interval
$\mathrm{Cp}=1.8 \mathrm{MHz}$ and Partial cancellation factor $=0.03$.

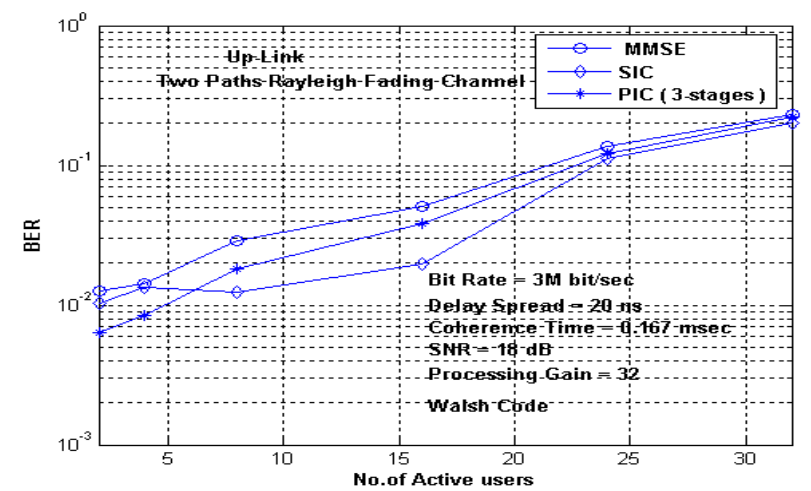

(a)

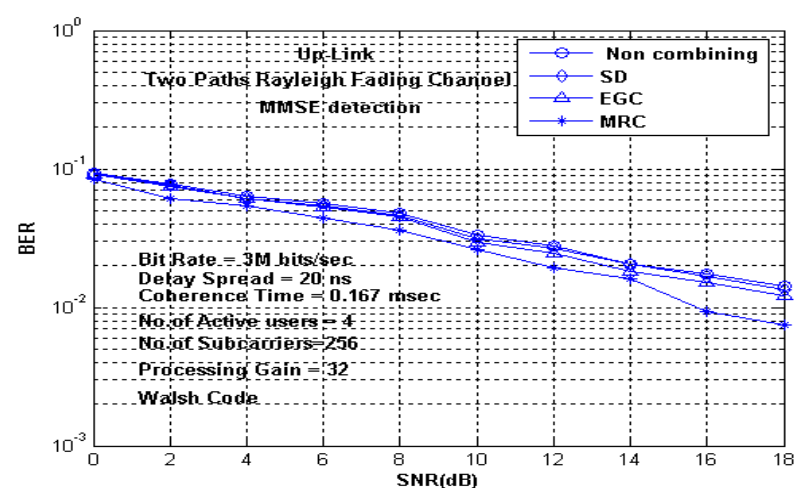

(b)

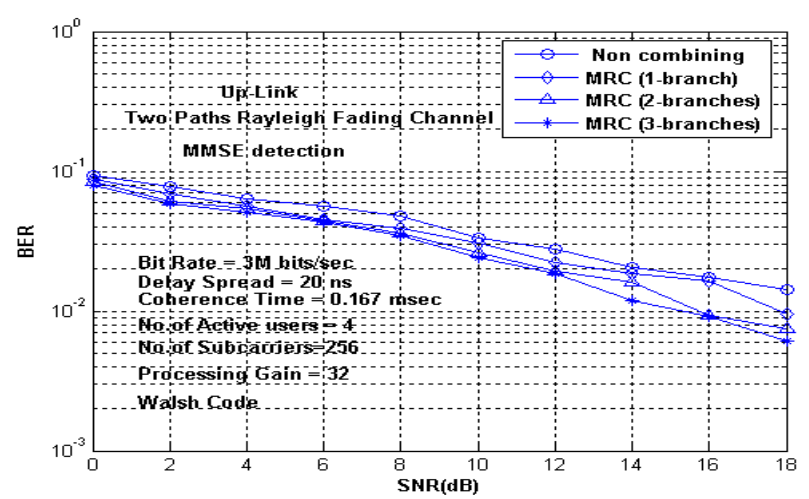

(c)

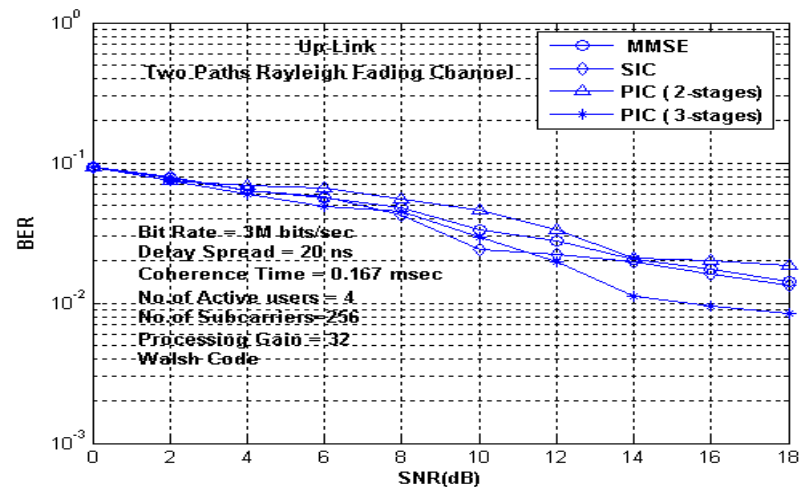

(d)

Figure 5. BER performance of GO-MC-CDMA system: a) versus number of active users, b) using different types of data detection techniques, b) for different diversity combining techniques, c) using different number of branches for MRC combining technique, d) with different types of data detection techniques 


\section{Conclusions}

Maximal ratio combining (MRC) takes better advantage of all the diversity branches in the system. A special case of MRC is presented in the next section. MRC always performs better than either selection diversity or equal gain combining EGC because it is an optimum combiner. The information on all channels is used with this technique to get a more reliable received signal[5].

For high number of active users, SIC detector is efficient because the number of stages is equal to the number of active users, but the time delay between the users is very high. PIC detector also has a good result when the number of active users is increased, and it becomes more complex for design. This criterion is shown in Figure 5a. Figure 5b shows the advantage of using different diversity combining techniques to improve bit error rate in GO-MC-CDMA system. The SD and EGC combining improve the bit error rate slightly as compared with non combining system.

MRC technique gives better BER performance as compared with SD and EGC combining diversity techniques, but it is more complex in the design.

\section{REFERENCES}

[1] Shinsuke Hara, Ramjee Prasad,"Overview of Multicarrier CDMA”, IEEE Communications Magazine, pp 126-133, Dec 1997

[2] Xiaodong Cai, Shengli Zhou, and Georgios B. Giannakis," Group-Orthogonal Multi-Carrier CDMA", IEEE

Transactions On Communications, 2003, pp 1-10

[3] Hafeth Hourani, "An Overview of Diversity Techniques in Wireless Communication Systems", Postgraduate Course in Radio Communications (2004/2005), Helsinki University of Technology

[4] Kai Dietze, "Analysis of a Two-Branch Maximal Ratio and Selection Diversity System with Unequal Branch Powers and Correlated Inputs for a Rayleigh Fading Channel", M.S.c thesis, Dept. of Electrical Engineering, Blacksburg, Virginia, March 30, 2001

[5] Hak Keong Sim,"Near Maximum Likelihood Multiuser Receivers for Direct Sequence Code Division Multiple Access", A thesis for the degree of Doctor of Philosophy. The University of Edinburgh, November 2000

[6] L.Hanso, M.Munster, B.J.Choi and T.Keller “ OFDM and MC-CDMA for Boroadband Multi User Communications, WLANs and Broadcasting" John Wiley and Sons, Ltd., England, 2003

[7] E. Costa, M. Midrio, and S. Pupolin, "Impact of nonlinearities on OFDM transmission system performance," IEEE Commun. Lett., vol. 3, no. 2, pp. 37-39, Feb. 1999

[8] H. Cheon and D. Hong, "Effect of channel estimation error in OFDM based WLAN," IEEE Commun. Lett., vol. 6, no. 5, pp. 190-192, May 2002

[9] J. Tellado, L. Loo, and J. Cioffi, "Maximum-likelihood de- tection of nonlinearly distorted multicarrier symbols by iterative decoding," IEEE Trans. Commun., vol. 51, no. 2, pp. 218-227, Feb. 2003

[10] H. Ochiai and H. Imai, "Performance analysis of deliberately clipped OFDM signals," IEEE Transactions on Communications, vol. 50, no. 1, pp. 89-101, 2002

[11] Luca Rugini, Paolo Banelli and Geert Leus " Reduced Complexity Equalization for MCCDMA systems over Time Varying Channels, Proc. of the ICASSP 2005, pp 473-487

[12] ETSI, "Digital Video Broadcasting (DVB): Framing structure, channel coding and modulation for digital terrestrial television", EN 300 744, V. 1.4.1, January 2001

[13] ETSI, "Radio broadcasting systems: Digital audio broadcasting (DAB) to mobile, portable and fixed receivers", EN 00 401, V. 1.3.2, September 2000

[14] A. Armada, "Understanding the effects of phase noise in orthogonal frequency division multiplexing (OFDM)", IEEE Transactions on Broadcasting, Vol. 47, No. 2, pp. 153-159, June 2001

[15] D. Petrovic, W. Rave, G. Fettweis, "Phase noise suppression in OFDM including intercarrier interference", International OFDM Workshop (InOWo), pp. 219-224, September 2003

[16] M. Gholami, S. Nader-Esfahani, A. Eftekhar, "A new method of phase noise compensation in OFDM", IEEE International Conference on Communications, ICC2003, pp. 3443-3446, May 2003

[17] V.S. Anishchenko, T.E. Vadivasova., G.A. Okrokvertskhov, G.I. Strelkova, "Correlation analysis of dynamical chaos", Elseiver, Physica A 325, pp. 199-212, 2003

[18] Slock, "Signal Processing challenges for wireless Communication," in Proc, 1st Int. Sym. on Control, Communications and Signal Processing, Tunisia, pp. 881-892, March 21-24 2004

[19] Coleri, S., Ergen, M., Puri, A., and Bahai, A., "Channel Estimation Techniques Based on Pilot Arrangement in OFDM Systems," IEEE Transactions on Broadcasting, vol. 48, pp. 223-229, Sept. 2002

[20] Wu, J., and Wu, W., "A Comparative Study of Robust Channel Estimators for OFDM Systems," Proceedings of ICCT, pp. 1932-1935, 2003

[21] Yang, B., Letaief, K. B., Cheng, R. S., and Cao, Z., "Channel Estimation for OFDM Transmission in Multipath Fading channels Based on Parametric Channel Modeling," IEEE Transactions on Communications, vol. 49, pp. 467-479, March 2001

[22] Hou, X., Li, S., Liu, D., Yin, C., and Yue, G., "On Two-dimensional Adaptive Channel Estimation in OFDM Systems," 60th IEEE Vehicular Technology Conference, Los Angeles, Ca., vol. 1, pp. 498-502, Sept. 2004

[23] Sanzi, F., Sven, J., and Speidel, J., "A Comparative Study of Iterative Channel Estimators for Mobile OFDM Systems," IEEE Transactions on Wireless Communications, vol.2, pp. 849-859, Sept. 2003

[24] Li, Y., "Simplified Channel Estimation for OFDM Systems with Multiple Transmit Antennas," IEEE Transactions on Communications, vol. 1, pp. 67-75, January 2002 
[25] Auer, G., "Channel Estimation in Two Dimensions for OFDM Systems with Multiple Transmit Antennas," GLOBECOM, pp. 322-326, 2003

[26] IEEE P802.16 (Draft 8, May 2005), WiMAX Specification

[27] Z. Li and M. L.aho, Nonblind and Semiblind Space-Frequency Multiuser Detection for Multirate MC-CDMA Systems, IEEE Transactions On Signal Processing, VOL. 54, NO. 11, NOVEMBER 2006, pp. 4393-4404

[28] J. Zhan, G. Liao and G. Li, A novel transmitter design for GLSFBC-OFDM-CDMA communication systems", Signal Processing 87,2007 , pp. 2251-2259

[29] M. Guenach and H. Steendam, Performance Evaluation and Parameter Optimization of MCCDMA, IEEE Transactions on Vehicular Technology, VOL. 56, NO. 3, MAY 2007 , pp. 1165- 1175

[30] M. El-Hajjar, B. Hu, L.L. Yang and L. Hanzo, Coherent and Differential Downlink Space-Time Steering Aided Genera- lised Multicarrier DS-CDMA, IEEE Transactions on Wireless Communications,, VOL. 6, NO. 11, NOVEMBER 2007. pp. 3857-3863

[31] T. Hwang, Ye. L. Yi Y.Wu, Energy Spreading Transform for Down-Link MCCDMA, IEEE Transactions on Wireless Communications, VOL. 7, NO. 5, MAY 2008, pp. 15221526

[32] W. M. Jang, L. Nguyen and M. W. Lee, MAI and ICI of Asynchronous Uplink MCCDMA With Frequency Offset, IEEE Transactions on Vehicular Technology, VOL. 57, NO. 4, JULY 2008, pp. 2164- 2179

[33] A. B. Djebbar, K. Abed-Meraim, and A. Djebbari, Blind and Semi-Blind Equalization of Downlink MC-CDMA System Exploiting Guard Interval Redundancy and Excess Codes, IEEE Transactions on Communications, VOL.57, NO. 1, Jan. 2009. pp. 156-163

[34] L. Hanzo, et al, Single and Multi-Carrier DS-CDMA, $1^{\text {st }}$ Edition, IEEE Press, Jon Wiley \&Sons, Chichester, 2003 